\title{
A Proof-of-Concept Visualization to Increase Comprehension of Personal Medication Schemes
}

\author{
Robin De Croon \\ Joris Klerkx \\ KU Leuven, Department of Computer Science \\ Celestijnenlaan 200A, B-3001 Leuven, Belgium
}

robin.decroon@cs.kuleuven.be joris.klerkx@cs.kuleuven.be erik.duval@cs.kuleuven.be

\begin{abstract}
In this paper, we investigate how information visualization techniques can be leveraged to increase patient comprehension of personal medication schemes in order to make it easier for them to explore, explain and understand drug information. Using computer vision techniques, our solution is able to recognize medication boxes, or so-called pharmaceutical packages, which are laid on an ordinary table. A projector visualizes drug information such as interactions, adverse drug reactions, intolerances and the dosage regimen around corresponding boxes. Five prototypes are designed and evaluated following a user-centered, rapidprototyping methodology. Test participants in our study included both general practitioners (GPs) and patients. Results are promising and clearly indicate that information visualization techniques are an effective means to explore and understand drug information. Even if this system was originally envisaged to be used as a means to improve 'therapy dialogue' between GPs and their patients during consultations, our results show that both GPs and patients think it would be highly beneficial if patients were able to use the system at home.
\end{abstract}

\section{CCS Concepts}

-Human-centered computing $\rightarrow$ Information visualization; Empirical studies in $H C I$;

\section{Keywords}

Information visualization; personal medication scheme

\section{INTRODUCTION}

General practitioners (GPs) have too much work [14]. Moreover, the resulting lack of time influences doctorpatient relationships and compromises patient-centered care [14]. We learned in previous meetings with GPs that they like patients who arrive prepared. However, health information is complex and is not always straightforward

Permission to make digital or hard copies of all or part of this work for personal or classroom use is granted without fee provided that copies are not made or distributed for profit or commercial advantage and that copies bear this notice and the full citation on the first page. To copy otherwise, to republish, to post on servers or to redistribute to lists, requires prior specific permission and/or a fee.

PervasiveHealth 2016, May 16-19, Cancun, Mexico

Copyright (๑) 2016 EAI 978-1-63190-051-8 to explore or communicate. The degree to which people have the capacity to obtain, process, and understand health information is called health literacy. We argue that information visualization technology can help to improve 'therapy dialogue' and thus make it easier for a GP to explain complex health information. More specifically, we wanted to learn if we can augment the practitioner's office by installing a system that is able to visualize health information on, for example, the table that is usually between the GP and the patient. The GP can then use these visualizations to discuss health information more efficiently with a patient.

Discussion groups were organized as part of a larger project which examines the potential added value of unlocking health data for patients and looks for solutions to bring down potential barriers in the patient-caregiver interaction. In each group patients elicited questions around medication. "Why do I have to take this medication?", "Which are my adverse reactions?", and "Are there any alternatives?" are examples of commonly asked questions. Drug information can be cumbersome after all: dosage regimen, adverse drug events, and interactions are only a few examples of information patients should understand concerning medication intake. In this paper we propose an information visualization technology approach as a potential solution to address this problem. Hence, the aim of this study is to determine if information visualization technology has the potential to increase patient comprehension of their personal medication schemes.

Based on lessons learned from related work and interviews with patients during the discussion groups, we designed a proof-of-concept that assists both patients and GPs to explore and discuss medication related information. More specifically, using computer vision techniques our solution recognizes medication boxes ${ }^{1}$ which are laid on an ordinary table. A projector visualizes drug information such as interactions, adverse drug reactions, intolerances and the dosage regimen around corresponding boxes as can be seen in Figure 1. The choice for medication boxes is made because patients are able to buy medication without prescription or even in a supermarket. This means that electronic medical records are not always up to date. It is thus hard to maintain an overview of the medication a patient is currently taking and expose possible unwanted interactions. To tackle this issue, GPs can ask patients to collect all their medication boxes and bring them to the office. Without the boxes patients would need to write down all medication they take

\footnotetext{
${ }^{1}$ Refers to the actual (paper) box that contains blister packs or bottles; also called pharmaceutical packages
} 


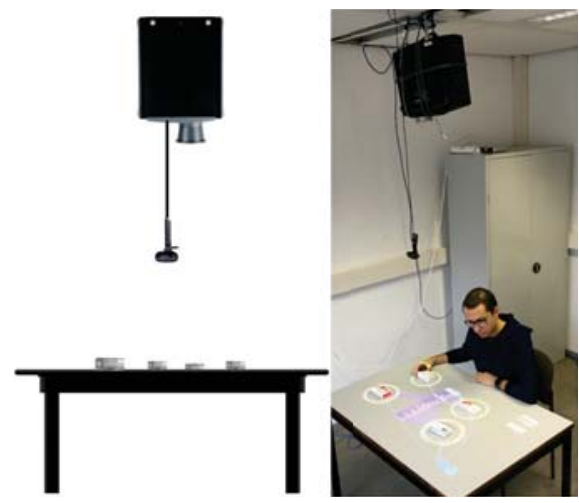

Figure 1: (left) Schematic side view of the set-up. (right) Real-life set-up of the final design with four real medication boxes.

and this is error prone due to hard spelling and variations of medication. Moreover, this set-up also supports patients who already take their medication to a GP.

\section{LITERATURE STUDY}

This section starts with an overview of research opportunities, discusses visualizations dealing with drug information and concludes with a discussion on tabletop affordances.

\subsection{Information Visualizations Opportunities}

Caban and Gotz [9] stated that "[t]he large volume of clinical data now being captured for each patient poses many challenges to clinicians trying to combine data from different disparate systems and make sense of the patient's condition within the context of the patient's medical history" [9]. A potential approach to deal with these large amounts of data are information visualizations. Health information visualizations can indeed provide opportunities to improve health care [21]. Hence, Shneiderman, Plaisant and Hesse [21] list three overlapping domains of what they call 'Health 2.0': first, visualizations should use personal information such as personal medication schemes; second, visualizations should extract data from an electronic medical record (EMR) such as the reason for taking a drug; and finally visualizations should use public health information such as a public database to look up possible adverse reactions. More specifically from an HCI approach, Nunes et al. [17] consider existing collaborations in self-care as one of the opportunities to improve the quality of care. Furthermore, visualizing chronic condition data emerged as one of their six design-related tensions from their analysis

Rxplore [15] is an example of a visual tool that helps GPs assess medication side-effects. Even though Rxplore only uses simple bar charts, it is able to retrieve information on multiple medications simultaneously. The second reported strength is the immediately interpretable visual representation of medical data. Furthermore, we can learn from Siek et al. [22] that there is a contradiction between caregivers, who prefer the overview of an entire day, and older adults who primarily want to see when their next medication was scheduled [22]. However, from their first study, we also learn that their participants liked the visual qualities of a clock visualization and kitchen counter prototypes [22]. Another valuable research project is the Visualizing Health project that contains 54 examples of tested visualizations. In comparative tests between tabular and non-tabular formats, graph 'Tables of side effect risks with icons ${ }^{\prime 2}$ helped viewers best to understand how different side effect vary in their risk and severity.

\subsection{Tabletop Affordances}

We will combine lessons learned from previous medication visualization research with tabletop affordances to increase patients' comprehension of personal medication schemes. Piper and Hollan [19] have shown that a large horizontal touch-screen suits the needs of older patients and that it can facilitate the doctor-patient interview process. They conclude that older adults like to have a shared view of electronic medical records and that "a large multiuser, multitouch display may be an effective device for presenting such information to older patients." [19]. This is in line with Asan et al. [4] who have found that active information sharing seems to engage patients and that training and design can help doctors to engage patients in communication. The optimal positioning of the computer screen has the potential of improving interpersonal communication between caregivers and patients [2]. However, in the health domain tabletop devices are mostly used to train physicians [24] or help patients rehabilitate [3]. Nevertheless, in the domain of learning analytics, tabletops have been extensively researched. Dillenbourg and Evans [13] provided an extensive overview of tabletop research in the learning analytics domain. However, these concepts can also be applied in the health domain as an expert user can be the teacher or the GP and the layman can be a student as well as a patient. In our work we apply their suggested affordances, such as hands-on activities, and design recommendations, such as the use of objects by projecting the proposed visualizations around medication boxes.

\section{ITERATIVE DESIGN PROCESS}

This section starts with a description of the chosen approach followed by a discussion of the initial design. Changes to the design are documented together with the motivation for each change in the subsequent subsections.

\subsection{User-centered, Rapid-prototyping Design}

In order to augment the GP's office with projected visualizations, a user-centered, rapid-prototyping methodology was applied to gradually improve the initial design of the visualizations. This approach has proven to be successful in other information visualization studies in the pervasive health domain $[12,17]$. In line with this methodology, our design evolved quickly. After every evaluation, qualitative feedback was addressed in the next prototype, which was then again evaluated. One low and four high fidelity prototypes were designed and evaluated.

Patients in general, and more specifically adults who need to take medication, were selected as the target audience. Participants were recruited through mail or a phone call, in which we shortly explained the goal of this research and asked if they would want to participate. Participation was voluntary and not compensated. Furthermore, they

${ }^{2}$ See http://www.vizhealth.org/gallery/assets/89/ for an example on how to represent side effects severity and risk. 


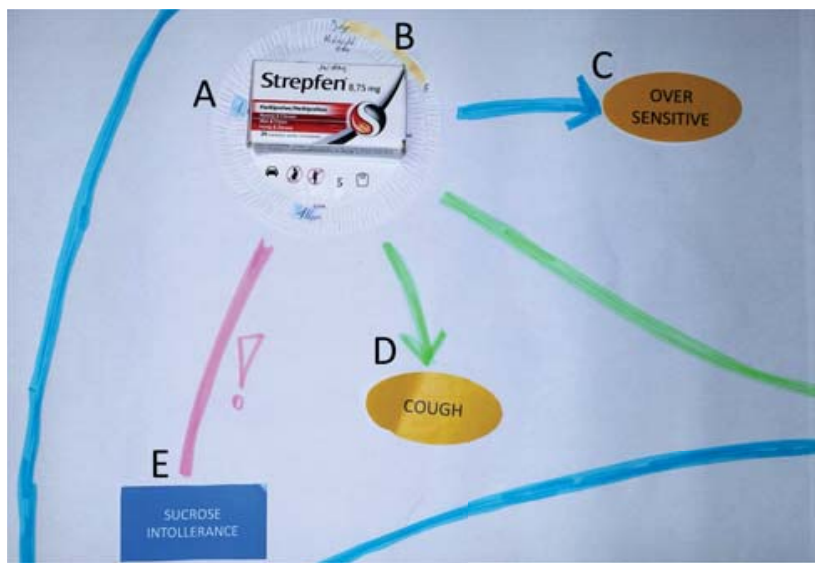

Figure 2: Part of the initial low fidelity prototype.

could only participate once. Throughout individual face-toface interviews, the concurrent Think-Aloud Protocol [16] was applied to let participants explain what they think when seeing the visualizations. In this way it can be tested whether participants understand the message the visualization tries to convey.

\subsection{Low Fidelity Iteration}

The first prototype, of which a small segment is shown in Figure 2, was designed on paper. Nevertheless, this allowed us to test the initial design where patients put their medication boxes on the table between them and a GP. Once the medication is put on the table, the system hypothetically recognized the boxes and visualized concentric circles surrounding the corresponding medication boxes. The inner circle visualizes the dosage regimen (Figure 2A) and is visualized as a 24-hour clock. The colored segments indicate between which hours patients should take medication. The outer circles are subdivided into three categories: 1) lifetime medication, visualized with one red circle; 2) time-independent or if-needed medication, visualized with one green circle; and 3) medication that should be taken during a certain period visualized using one or more (for each month) orange concentric circles (Figure 2B). An auxiliary text in the upper part of each circle explains which month each circle represents. GPs can choose which medication related information they want to discuss and can put a paper card on the table for each adverse reaction (Figure 2C), episode (Figure 2D), and allergy/intolerance (Figure 2E) of the patient. A network is drawn to link common elements after the boxes are recognized. Green arrows for reasons and/or linked episodes, blue arrows for adverse reactions and red arrows for interactions between medication or between medication and allergies.

The initial design was tested with five participants. Since the low fidelity prototype was made on paper, a researcher acted as the computer that recognizes the boxes and draws the projected interface. Furthermore, six medication boxes, that were not necessarily familiar to the participant, were provided by the researcher so the interface could be drawn in advance in order to save time. Hence, the low fidelity prototype was only tested with these boxes. Nevertheless, these six boxes were sufficient to test the initial design. The evaluation protocol as explained in Section 3.1 was applied when the participant interacted with the system.

Only two out of five participants correctly linked the green color to episodes and blue colors to adverse reactions. Moreover, participants linked these colors to the colors used in the circles surrounding the boxes. Another confusion for three participants was the colors used to separate between lifetime and if-needed medication. Participants perceived a green color as safe to take, whereas a red colors indicates danger. However, in the initial design medication that can be taken if needed is shown in green and a lifetime medication red. Finally, two participants rightfully stressed out the fact that there are too many possible adverse reactions for GPs to put cards on the table. Nonetheless, the initial design was a good start. However, these remarks needed to be addressed in the next prototype.

\subsection{First High Fidelity Iteration}

The high fidelity prototype was designed based on the lessons learned as described in the previous section. The goal of this iteration was again to evaluate the concept and discover possible usability issues. Furthermore, it was tested whether the three changes implemented were successful: 1) Only one color is used for the arrows as the direction indicate the reason for taking a drug (towards the medication box, (Figure 3A) and possible adverse reactions (towards the adverse reaction, (Figure 3B). 2) Episodes and adverse reactions are no longer individual cards, but are a computer generated list on the left side of the projection area. 3) If-needed medication is drawn with only the dosage regimen circle (Figure $3 \mathrm{C}$ ) and lifetime medication is drawn with one green circle, which is the opposite color of the paper prototype. After all, the system did not want to give the impression a medication patients have to take their entire life is 'bad'. Other medication was still visualized using orange circles as shown in Figure 3D.

This updated design was also tested with five participants using the same evaluation protocol. All participants liked the overview the system provides, the opportunity to move boxes around to create their personal view, and the clear overview of possible adverse reactions. However, three out of five participants did not immediately understand the outer circles represented months. It became clear that using full circles to represent months is not optimal since each day starts at a slightly different angle for a 30- or a 31day month. Three out of five participants explained they would understand the design better with more auxiliary text. Two out of five participants were confused with the start of each arrow. Since each arrow started from the outer circle, two participants thought risks are only relevant at that particular moment the arrow originated. Furthermore, arrows were drawn without collision detection. Hence, two participants mentioned the visualization was too crowded; especially when medication with numerous potential adverse reactions was put on the table. Although this prototype was an improvement over the initial design, previously mentioned usability remarks needed to be addressed in an additional prototype.

\subsection{Public Iteration}

The third prototype was based on the lessons learned in the first high fidelity prototype. We wanted to test whether new changes were successful: 1) The concentric 


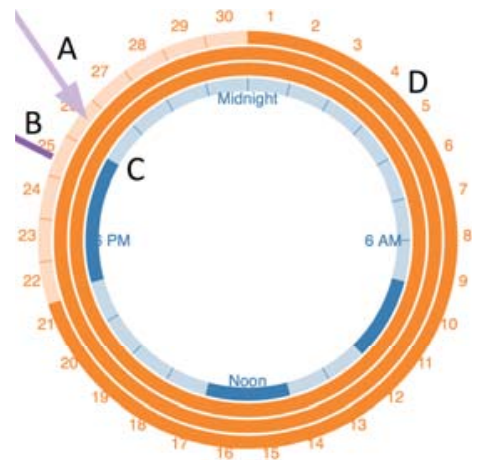

Figure 3: A visual example of how circles were projected surrounding a medication box in the high fidelity iteration.

circles as shown in Figure 3 were altered to large concentric arc segments as shown in Figure 4A. In this visualization, each day is an arc of 11.4 degrees and starts at the same angle. There was thus still room for auxiliary text such as the name of each month. For example, November is an arc of 341 degrees and December of 352 degrees, see Figure 4A for a visual example. 2) Auxiliary text was added to differentiate between episodes, adverse reactions and personal data. 3) Lines were drawn from the center of each circle to avoid that users link adverse reactions to a time period as shown in Figure 4B. 4) The table colored red to warn users of a potentially dangerous situation when an interaction is detected.

We had the opportunity to test this third prototype as a fully functional proof-of-concept during the national company discovery day at the Computer Science department in Leuven. We provided ten medication boxes that could be used during the demo. The prototype was explained by a researcher in approximately one minute. In total 220 persons interacted with the demo and stayed on average almost 5 minutes (288s, $\sigma=143 \mathrm{~s})$. Participants expressed their interest by asking when they could actually start using the system in a personal setting. Even though not scientifically relevant, people were interested if there were plans to commercialize the system, which stresses the genuine interest of people and the need for such a system.

Three users, who indicated they have to take medication on a regular basis, wanted to be informed of cheaper alternatives. Four care providers indicated it would be useful to visualize the probability of adverse reactions. Unfortunately, some users experienced problems following the lines. Especially when a medication box was placed on the right side, which is the opposite side of the episodes and adverse reaction. Therefore, lines could be long and hard to follow around several other medication boxes. The changes made in this prototype performed well. Only minor changes needed to be implemented in the next iteration. Nonetheless, feedback from GPs needed to be gathered before the visualizations could be finalized.

\subsection{Medical Iteration}

This iteration served to gather extensive feedback from GPs. We primarily wanted to test the medical relevance and learn if the visualizations are clear to GPs as well. As with the previous iterations, changes as a result of user

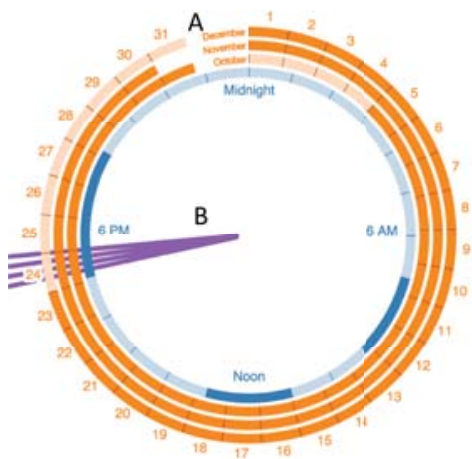

Figure 4: Updated visual example of how circles were shown in the public iteration.

feedback were implemented in a fourth prototype and tested: 1) Using a five category frequency scale ${ }^{3}$ adverse reaction probability can be classified as ordinal data. To help the user judge on this information color saturation was used. 2) Since the color scheme from the second prototype was not sufficient anymore a new color scheme was mapped out. Negative interactions were colored red as the system was tested in the Western culture. To maintain consistency, adverse reactions and episodes were drawn in analogous colors: purple for adverse reactions and blue for episodes. In addition, labels were colored similarly to aid user's short term memory to quickly recognize the meaning of each color. To visualize time information complementary colors were used: the dosage regimen in yellow, the schedule in orange and lifetime medication in green. 3) Moreover, the rectangles were projected in the middle of the visualization instead of the left side. This way, lines were shorter and easier to follow. These colors are also used in the final design as is shown in Figure 5.

The system was evaluated for perceived usefulness using printouts and examples shown on a computer screen. Two expert GPs evaluated the system using the same evaluation protocol as in the first two iterations. Furthermore, they were asked specific questions such as "what kind of medication questions patients ask the most" and "what do you experience is hardest to explain?". As expected, answers were in line with questions asked by patients.

The design was well received. The major concern raised was time pressure during a consultation. Therefore, the GPs argued the system should be made accessible to patients before they visit. This way questions the patient might have can be solved autonomously using the system. Unclear answers can then be discussed afterwards during a face-toface meeting using the system. It must also be possible for GPs to configure the level of adverse reactions shown due to the nocebo phenomenon [6]. In contrast, patients expressed they liked the fact that they have the same overview as their GP. Concerns were also raised that adverse reactions are rarely registered. Furthermore, the source of each data element should be unambiguous. Finally, it was

${ }^{3}$ The Council of International Organizations of Medical Sciences divided the frequency of adverse drug reactions in five categories: 1$)$ very common $(>=1 / 10), 2)$ common $(>$ $=1 / 100$ and $<1 / 10), 3)$ uncommon $(>=1 / 1000$ and $<$ $1 / 100)$, 4) rare $(>=1 / 10000$ and $<1 / 1000)$, and 5) very rare $(<1 / 10000)$. 
brought to the researchers attention that food can also cause interactions [10]. The feedback will be incorporated into the final design.

\subsection{Final Design}

The final design is shown in Figure 5 and is the result of all lessons learned thanks to the user-centered, rapidprototyping approach. One minor visual update is added to the previous iteration. The source of data is made explicit as this was a requirement of the GPs. Data originating from the EMR is displayed on the left (Figure $5 \mathrm{~A}+\mathrm{C}$ ), data from other trusted sources, such as adverse reactions that are retrieved from a validated database, remain in the middle (Figure 5B) and patient added data such as food could be visualized on the right.

\section{TECHNICAL DETAILS}

The system to recognize the medication boxes is implemented using JavaCV ${ }^{4}$. To recognize the medication boxes a Logitech c270 with a 1280x960 resolution and a 60 degrees field of view was used in the first high fidelity prototype and a Logitech c930e camera with a 1920x1080 resolution and a 90 degrees field of view in the subsequent iterations. Furthermore, a NEC MT1055 projector with a $1024 \times 768$ resolution was used to project the first high fidelity prototype on a $120 \mathrm{~cm}$ x $80 \mathrm{~cm}$ x $75 \mathrm{~cm}$ table. In the subsequent prototypes an Epson EH-TW5000 with a 1920x1080 resolution projected the visualizations. The visualization itself, which is projected around medication boxes, is implemented using the D3js [7] library. Episodes, allergies and personal data are extracted from an EMR using simple SQL queries, while adverse reactions are manually extracted from medication leaflets. However, during the user tests a modified patient record was used for anonymity.

In order to improve the implementation, an additional technology iteration was organized after the second iteration with five participants to discover potential bugs. In this iteration, a Samsung UE60F6100 TV is used instead of a projector. We wanted to test if hanging the camera closer to the table, could improve the accuracy of the detection and thus tighten the virtual bounding boxes surrounding each medication package. However, the TV performed worse than expected since light emitted by the TV darkens the top of the medication boxes the camera needs to detect. On the other hand, with a black background, reflections from the lights in the ceiling were an issue. Furthermore, due to the relatively low resolution of the TV for this large working environment, text was unreadable. Finally, the field of view limited the height of the camera.

\section{FINAL EVALUATION}

The goal of the final evaluation was to determine if information visualization techniques are an effective means to help increase patients' comprehension of their personal medication schemes. Furthermore, the evaluation also served to assess the perceived usefulness and usability of the last design.

\subsection{Evaluation Protocol}

The final design was evaluated with 25 participants (16 males and 9 females) who were on average $32(\sigma=16.3)$

\footnotetext{
${ }^{4}$ https://github.com/bytedeco/javacv
}

years old; their age ranging from 18 to 75 years. Participants were recruited in a similar manner as described in Section 3.1. Eleven participants needed to take medication on a daily basis, while only one participant visited his GP every month. Participants were asked to fill in a short questionnaire measuring relevance and interest. Thereafter they were asked to perform a list of tasks (which will be discussed in further detail in Section 5.2) on a medication scheme with four different medications. These tasks were reviewed beforehand by a GP to test medical relevance of each task. Furthermore, time-to-task and the number of errors was measured. As with the evaluations in the iterative design process, participants were asked to think aloud [16]. Moreover, they were not recorded. The evaluation ended with six questions to measure if the visualizations were clear (green and blue box plots in Figure 6), five perceived usefulness [18] questions (purple box plots in Figure 6), and a System Usability Score (SUS) [8] questionnaire. The interactive version installed at the department was shown to 14 participants, whereas 11 participants were shown a static version as it was not always possible to install the entire system at their homes.

\subsection{Usability and Tasks}

The system scored an average SUS score [8] of 79.5 $(\sigma=10)$. This high usability score reflects on the timings participants needed to perform each task and on the low number of mistakes made. The first task where participants were asked "When do you have to take drug 1?" took on average $16 \mathrm{~s}$ ( $\sigma=9.4,1$ error). Tasks where they only needed to follow a line were completed with an average time less than 10s: "Why do you have to take drug 2?" $(9 \mathrm{~s} \pm 11.7 \mathrm{~s}, 1$ error), "Which are all the possible adverse-reactions of drug 1 ?" (7s $\pm 4.8 \mathrm{~s}, 0$ errors), "Can you currently drive a car?" (9.8s $\pm 12.1 \mathrm{~s}, 3$ errors), "Can you still breastfeed your child?" $(3.2 \mathrm{~s} \pm 3.3 \mathrm{~s}, 0$ errors $)$. Other tasks were completed in less than 15s: "What is the probability that you will catch a dry throat?" (12.8s $\pm 10.8 \mathrm{~s}, 0$ errors), "Can you combine drug 1 and drug 3?" (10.8s $\pm 7.7 \mathrm{~s}, 2$ errors), "At what times do you have to take drug 2?" (10.1s $\pm 11.3 \mathrm{~s}, 1$ error $)$.

\subsection{Likert Scale Questionnaires}

As illustrated in Figure 6, answers to Likert scale questions scored consistently. When participants were asked if our system increased their comprehension of medication schemes all but one participant (neutral) agreed; $64 \%$ participants even strongly agreed. Furthermore, participants agreed that the design can create medication awareness and as well as that there is a need for a system to help explore drug information. The six blue box plots in Figure 6 concern dialogue-related questions. Participants strongly agreed our design can indeed help GPs explain personal medication schemes. On top of that, they agreed they would actually like to use the system when they discuss medication with their GP. On the other hand, only $76 \%$ participants agreed the system can help them to explain their medication. Although the system was originally envisaged to be used as a means to improve the dialogue between GPs and their patients during consultations, $84 \%$ (the other $16 \%$ was neutral) indicated they do not need a GP to use the system. In fact, $80 \%$ participants preferred to use the system at home. Whereas only three participants preferred the pharmacy, one participant preferred the GP's practice 


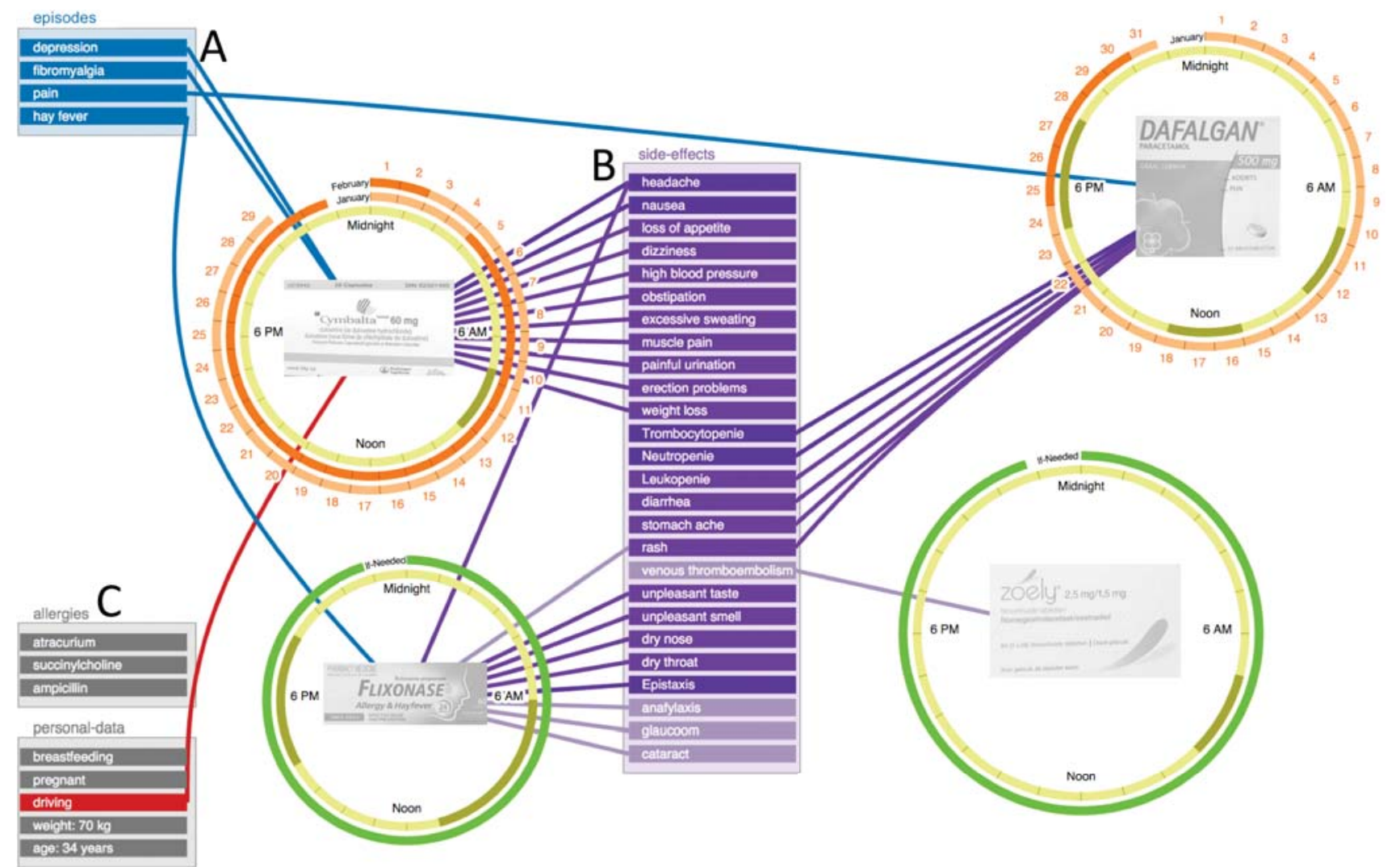

Figure 5: Overview of the final design. Blue rectangles and lines visualize the reasons why a patient has to take each particular medication (A). The lower right circle does not have an EMR registered reason and can be a drug the patient bought without prescription. Purple rectangles and lines visualize possible adverse reactions, while a higher saturation indicates a higher frequency of occurrence (B). In the lower left corner personal data such as allergies and age are drawn to visualize interactions using red lines (C). In this figure, the medication images are added afterwards to illustrate how the visualization looks when projected around the boxes, in reality these are real medication boxes as is also visible in Figure 1.

and one participant wanted to use the system at all three locations. Finally, the four purple box plots in Figure 6 show the distribution on the perceived usefulness questions. Participants agreed this was the right kind of tool to explore drug info and that the system provided a useful source of feedback. This approach could help to prevent medical mistakes according to $84 \%$ ( $12 \%$ neutral and $4 \%$ disagree). Finally, the details were at the right level as reported by $84 \%$ ( $8 \%$ neutral, $8 \%$ disagree) of participants.

\section{DISCUSSION}

Overall, the results of this study indicate the system can indeed help to increase patient comprehension of their personal medication schemes. Moreover, even if this system was originally envisaged to be used as a means to improve 'therapy dialogue' results show that both GPs and patients think it would be highly beneficial if patients were able to use the system at home.

\subsection{Iterations}

Changes made to the initial design, as described in Section 3, improved functionality and general usability. In all iterations, participants only raised usability issues

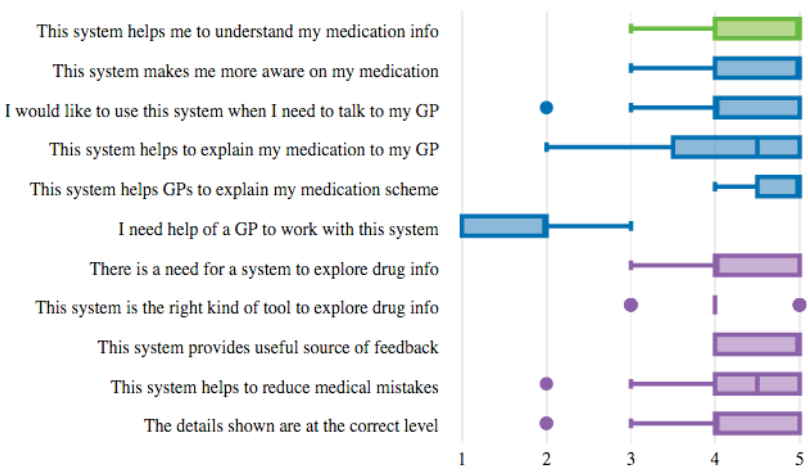

Figure 6: Answers ranging from 1 (strongly disagree) to 5 (strongly agree). The green box is related to our research question, blue boxes indicate dialogue related questions and purple boxes visualize perceived usefulness questions. 
and additional requirements, none disagreed with decisions made. Suggestions raised during the different iterations were incorporated. However, patients and GPs disagreed on the level of detail shown. Patients mentioned they like the fact that they have the same overview, yet GPs expressed the necessity of being able to configure the level of details shown to the patient for health reasons, such as the nocebo phenomenon [6]. This is a complex issue with implications on how the patient can understand and manage their condition [17]. As our design requires uptake from GPs first, we currently follow the GPs' view and implemented programmatic flags that should be configured before the start of the system. This enables GPs to choose the level of details, such as the frequency of adverse reactions, shown to the patient. Furthermore, this does not interfere with Shneiderman's mantra [20] as all information that can be disclosed is still visualized in an overview.

\subsection{Final Evaluation}

The first task, where participants were asked to indicate on which days they had to take a particular medication, clearly took the longest time with an average duration of $16 \mathrm{~s}$. This may be due to the fact that this was the first task. On the other hand, this was also a harder task since participants needed to figure out that each orange circle represented one month. An initial training task would have enabled us to provide a clearer answer why this task took most time. The only qualitative feedback participants provided during this task was that is was not always immediately clear which was the current day. Although all other tasks were completed in less than $15 \mathrm{~s}$, some participants initially tried to form an answer based on adverse reactions. For example, when participants were asked if they could combine two medications, nine participants first checked the possible adverse reactions. However, in the end only two participants made a mistake by drawing their conclusion on adverse reactions and not on the visualization. We noticed a similar pattern when participants were asked if they could still drive a car; six participants initially looked at the adverse reactions. Other situations where it occasionally took participants more than 30s to complete a task are because of older participants who needed their reading glasses first. Nevertheless, timings are shorter compared to the traditional way of opening the leaflet, find your own language, and find the right section. When we asked five participants to look up the adverse reactions in the leaflet it took them on average more than half a minute $(38 \mathrm{~s} \pm 8 \mathrm{~s})$. A technical solution can provide a faster solution, as is evident from our results.

The SUS score of 79.5 indicates the usability can be considered between 'good' and 'excellent' [5]. Yet, it could potentially be higher. The first question which asked if participants think they would use the system frequently scored on average only $3.92(\sigma=0.9)$ out of the five possible points. However, qualitative feedback learned us that users would only use the system when they actually have medication questions.

\subsection{Tendency to Consultation Preparation}

A common trend, that was already noticed during the iterative design process, is that participants were genuinely surprised by how many questions could be answered without the assistance of a GP. This reflects in the results in the final evaluation where $80 \%$ of participants indicated they would like the system at home and do not need help of a GP. On top of that, GPs indicated they do not have enough time for an in depth discussion with all patients. Moreover, using our design, participants were able to answer the questions as described in Section 5.2. Hence, thanks to this study, we learned both GPs and participants preferred to use our visualization individually. We, therefore, argue for a medication system included in personal health record systems that can be used to explore medication questions before a consultation. The proposed system can subsequently be used to explore and discuss nonresolved questions with a GP. However, our visualizations were not designed nor evaluated to be used on an everyday basis and it is not well known what it means to be in need of medication management support in everyday life [11]. Furthermore, people have divergent ways they want to integrate disease management into their homes [1]. It should thus be researched further if this individually gained medication comprehension actually leads to empowerment, or to confusion and anxiety [23].

\subsection{Limitations}

Four limitations of the present study should be acknowledged. First, although it is designed to be an interactive system, detection of medication boxes did only occur in near real-time. Approximately one second per box is needed to detect a box on the table. However, as this work primarily focuses on the perceived usefulness of our proof-of-concept, we did not measure efficiency parameters, such as response time. This might have influenced our results since participants could be reluctant to move medication boxes around for better readability. This is presumably why we could not detect any differences between the static and interactive version in the final evaluation. Nevertheless, participants still perceived the system as useful. Secondly, since this work describes a proof-ofconcept design, it is not evaluated 'in the wild'. Nonetheless, with this methodology we were able to demonstrate the perceived usefulness. Third, the system was not directly compared to other systems. The setup of this paper was primarily to evaluate if visualization technology can help to increase patient comprehension of their personal medication schemes. However, a direct comparison with other systems would have provided a stronger result. Finally, this paper did not take privacy concerns into account that could constraint the use of the system.

\section{CONCLUSION AND FUTURE WORK}

The PervasiveHealth domain has many stakeholders and perspectives are varied [17]. This paper used the strength of the HCI lens [17] and uses qualitative methods to determine the perceived usefulness of our system. Our proof-of-concept was designed following a user-centered, rapid-prototyping methodology and evaluated during five iterations as was observed earlier in Section 3. It was evaluated with in total 40 participants; of which 25 in the final evaluation. Moreover, the system was shown to 220 persons during the department's open house day.

Results are promising and clearly indicate that information visualization techniques are an effective means to explore and understand drug information. Moreover, our results suggest there is actually a need for a visual system 
to explore medication information. On top of that, our proof-of-concept is able to show useful feedback and can help both patients and GPs to explore adverse reactions, schedules and recognize dangerous interactions. Although our system was initially designed to be installed at the GP's office, results show that both GPs and patients think it would be highly beneficial if patients were able to use the system at home. In future work, we will focus on how to integrate our visualization into a personal health record that is linked to the GP's EMR. This could enable patients to explore medication questions individually before asking nonresolved questions to their GP with the help of our system. It is important our design is not just ported, but that specific affordances of each medium are researched. For example, in a personal health record medication system, the camera of a smartphone could be used to recognize medication or to scan barcodes.

We believe that with our iterative design process and final evaluation, we were able to show the perceived usefulness. This work will guide our future work, but it will hopefully also guide other researchers' future work, such as augmented reality medication systems using, for instance, smartglasses or IKEA's kitchen concept 2025.

\section{ACKNOWLEDGMENTS}

MyHealthData is a project co-funded by iMinds, a digital research institute founded by the Flemish Government. Project partners are EBMPracticeNet, CEBAM, HealthConnect, CM, and MindBytes with project support from IWT. The authors would also like to thank all participants and the anonymous reviewers for their extensive feedback to improve the quality of the paper.

\section{REFERENCES}

[1] R. Aarhus and S. A. Ballegaard. Negotiating Boundaries: Managing Disease at Home. In Proc. CHI, page 1223, New York, USA, 2010. ACM Press.

[2] F. Ajiboye, F. Dong, J. Moore, K. J. Kallail, and A. Baughman. Effects of Revised Consultation Room Design on Patient-Physician Communication. HERD Journal, 8(2):8-17, jan 2015.

[3] M. Annett, F. Anderson, D. Goertzen, J. Halton, Q. Ranson, W. F. Bischof, and P. Boulanger. Using a multi-touch tabletop for upper extremity motor rehabilitation. In Proc. OZCHI, page 261, New York, USA, 2009. ACM Press.

[4] O. Asan, H. N. Young, B. Chewning, and E. Montague. How physician electronic health record screen sharing affects patient and doctor non-verbal communication in primary care. Patient Educ Couns, 98(3):310-316, mar 2015.

[5] A. Bangor, P. Kortum, and J. Miller. Determining what individual SUS scores mean: Adding an adjective rating scale. JUS, 4(3):114-123, 2009.

[6] A. J. Barsky, R. Saintfort, M. P. Rogers, and J. F. Borus. Nonspecific Medication Side Effects and the Nocebo Phenomenon. JAMA, 287(5):622, feb 2002.

[7] M. Bostock, V. Ogievetsky, and J. Heer. D3 data-driven documents. IEEE Trans. Vis. Comput. Graphics, 17(12):2301-2309, 2011.

[8] J. Brooke. SUS - A quick and dirty usability scale. Usability evaluation in industry, 189(194):4-7, 1996.
[9] J. J. Caban and D. Gotz. Visual analytics in healthcare - opportunities and research challenges. $J$. Am. Med. Inform. Assoc., 22(2):260-262, 2015.

[10] A. Dahan and H. Altman. Food-drug interaction: grapefruit juice augments drug bioavailability mechanism, extent and relevance. Eur. J. Clin. Nutr., 58(1):1-9, jan 2004.

[11] L. G. Dalgaard, E. Grönvall, and N. Verdezoto. Accounting for medication particularities: designing for everyday medication management. In Proc. PervasiveHealth, pages 137-144. ICST, 2013.

[12] R. De Croon, J. Klerkx, and E. Duval. Design and evaluation of an interactive proof-of-concept dashboard for general practitioners. In Proc. ICHI, Dallas, TX, USA, 2015. IEEE.

[13] P. Dillenbourg and M. Evans. Interactive tabletops in education. Int. J. Comp-Supp Coll, 6(4):491-514, dec 2011.

[14] N. Doran, F. Fox, K. Rodham, G. Taylor, and M. Harris. Lost to the NHS - Why GPs leave practice early : a mixed methods study. Br J Gen Pract, 2016.

[15] J. D. Duke, X. Li, and S. J. Grannis. Data visualization speeds review of potential adverse drug events in patients on multiple medications. J Biomed Inform, 43(2):326-331, apr 2010.

[16] C. Lewis. Using the "thinking Aloud" Method in Cognitive Interface Design. Research report. IBM T.J. Watson Research Center, 1982.

[17] F. Nunes, N. Verdezoto, G. Fitzpatrick, M. Kyng, E. Grönvall, and C. Storni. Self-Care Technologies in HCI: Trends, Tensions, and Opportunities. ACM Trans. Comput.-Hum. Interact., 22(6):1-45, dec 2015.

[18] P. O'Leary, N. Carroll, and I. Richardson. The Practitioner's Perspective on Clinical Pathway Support Systems. In Proc. ICHI, pages 194-201, Verona, Italy, 2014. IEEE.

[19] A. M. Piper and J. D. Hollan. Supporting medical communication for older patients with a shared touch-screen computer. Int J Med Inform, 82(11):e242-e250, nov 2013.

[20] B. Shneiderman. The eyes have it: a task by data type taxonomy for information visualizations. In Proc. IEEE Symposium on Visual Languages, pages 336-343. IEEE Comput. Soc. Press, 1996.

[21] B. Shneiderman, C. Plaisant, and B. W. Hesse. Improving Healthcare with Interactive Visualization. Computer, 46(5):58-66, may 2013.

[22] K. A. Siek, D. U. Khan, S. E. Ross, L. M. Haverhals, J. Meyers, and S. R. Cali. Designing a Personal Health Application for Older Adults to Manage Medications: A Comprehensive Case Study. J Med Syst, 35(5):1099-1121, 2011.

[23] C. Storni. Multiple Forms of Appropriation in Self-Monitoring Technology: Reflections on the Role of Evaluation in Future Self-Care. Int J Hum Comput Interact, 26(5):537-561, apr 2010.

[24] U. von Zadow, S. Buron, T. Harms, F. Behringer, K. Sostmann, and R. Dachselt. SimMed: Combining Simulation and Interactive Tabletops for Medical Education. In Proc. CHI, page 1469, New York, New York, USA, 2013. ACM Press. 